\title{
Una Nueva Plaga Potencial de Papa en Argentina, Maecolaspis bridarollii (Bechyné) (Coleoptera, Chrysomelidae, Eumolpinae)
}

Nora Cabrera ${ }^{1}$, Héctor A Awarez C., ${ }^{2}$ Alicia N. López; ${ }^{2}$ Ana Vincin ${ }^{2}$

\section{Resumen}

En muestreos realizados en cultivos de papa en el sudeste de la provincia de Buenos Aires (Argentina), se registró la presencia de un crisomélido eumolpino del género Maecolaspis (Bechyné) que ocasionaba severos daños en los tubérculos. Los objetivos de este trabajo fueron: identificar al adulto, aportar datos sobre su bionomía y características del daño que produce. Como resultado se cita por primera vez a Maecolaspis bridarollii (Bechyné) en este cultivo; se redescribe y se aportan nuevos caracteres discriminatorios. Se observó que, a partir de mediados de marzo, las larvas que alcanzaron un mayor desarrollo, dañan los tubérculos, haciéndoles perforaciones profundas que desmejoran su calidad comercial.

Palabras claves adicionales: $\quad$ genitalia macho y hembra, piezas bucales, papa, M. scintilifiera.

\section{New Potential Potato Pest in Argentina, Maecolaspis bridarollii (Bechyné) (Coleoptera, Chrysomelidae, Eumolpinae)}

\section{Summary}

A chrysomelid of the genus Maecolaspis (Bechyné) was identified as the damaging agent to potato crops in samples obtained from the southeast of Buenos Aires Province (Argentina). The objectives

Aceptado para publicación: abril 1997.

1 Dra. en Ciencias Naturales, Departamento Científico de Entomología.

Facultad de Ciencias Naturales y Museo UNLP, Paseo del Bosque. (1900)

La Plata, Argentina.

2 Lic. en Zoología. Cátedra Zool. Agrícola. Facultad de Ciencias Agrarias. U.N. Mar del Plata, C.C. 276 (7620) Balcarce, Argentina. 
of this research were to identify the adult insect and produce information on its bionomics and on the characteristics of the damage it causes.

For the first time Maecolaspis bridarollii (Bechyné) is reported affecting potatoes. According to results, the best harvesting time to reduce damage was immediately after the potato crop completed its growth cycle, henee limiting exposure to the attack of mature $M$. bridarollii larvae on potato tubers. From mid-March the larvae that had reached full development bored into the tubers causing severe damage and affecting the commercial value of harvested potato tubers.

Additional index words: male and female genitalia, mouth parts, Maecolaspis scintillifera.

La papa (Solanum tuberosum L.) constituye uno de los cultivos más importantes de la producción agrícola argentina, siendo el sudeste de la provincia de Buenos Aires la zona productora de papa por excelencia. De acuerdo con estimaciones recientes, con un área plantada a nivel nacional de 103,305 ha, se produjo en el sudeste bonaerense el $40 \%$ de la producción total del país con solamente 42,000 ha sembradas (15).

Entre los insectos subterráneos de importancia económica en el cultivo de papa en esta región, se determinó la presencia de un crisomélido eumolpino perteneciente al género Maecolaspis Bechyné. El principal daño a las plantas, es provocado por las larvas que atacan los tubéculos haciéndoles perforaciones profundas que afectan su calidad original e impiden la competencia del producto argentino en mercados extranjeros.

El género Maecolaspis, endémico de América, fue creado por Bechyné (3) y está integrado por aquellas especies descritas antiguamente en el género Colaspis Fabricius. Varios representantes de Maecolaspis fueron citados sobre papa en la región neotropical $(1,11,14)$. En Argentina, si bien son 30 las especies presentes, sólo Maecolaspis chlorites (Erichson) fue mencionada atacando Solanáceas cultivadas: papa, berenjena (Solanum melongena L.), ají (Capsicum frutescens L.) y silvestres: granadillo (S. bonariense L.) y duraznillo (S. glaucum Dun.) (10). 
Esta especie presente en Bolivia, Perú y Brasil (Amazonas), ha sido incorrectamente citada para nuestro país (10). Este error se debe a que, frecuentemente presentan coloración y punteado elitral similar lo que dificulta su identificación.

En virtud de su importancia como plaga y la dificultad de su correcta identificación, los objetivos de este trabajo fueron: identificar y redescribir al adulto de $M$. bridarollii, con el aporte de nuevos caracteres discriminatorios: piezas bucales, nerviación alar, genitalia masculina y femenina e informar sobre su bionomía y características del daño que produce.

\section{Materiales y Métodos}

Los adultos y larvas se registraron mediante muestreos efectuados en una pastura y en un cultivo de papa, ubicados en la Estación Experimental Agropecuaria (E. E. A.) del INTA Balcarce $\left(37^{\circ} 45^{\prime} \mathrm{S}, 58^{\circ} 18^{\prime} \mathrm{O}\right)$ en la provincia de Buenos Aires, Argentina. En la pastura, cada muestra consistió en un bloque de tierra de $0.316 \times 0.316 \times 0.450 \mathrm{~m}$, y en el cultivo, la planta con la tierra que la rodeaba. Cada muestra se colocó en una bolsa de plástico y se llevó al laboratorio para su análisis. La tierra se desmenuzó y tamizó con un tamiz provisto de una red de acero de $1 \times 1 \mathrm{~cm}$ de malla, y se efectuó la extracción manual de los ejemplares en sus diferentes estados de desarrollo (larva, pupa, adulto). El material examinado para la identificación de los adultos provino de larvas criadas en el laboratorio, de recolecciones realizadas desde 1991 sobre las hojas de las plantas de papa, y de ejemplares depositados en las colecciones del Museo de La Plata (MLP). Con un ocular micrométrico adaptado a un microscopio estereoscópico se tomaron las siguientes medidas del cuerpo: largo total, ancho del protórax, largo del protórax y ancho máximo, tomados entre los puntos extremos y expresados en milímetros y se estableció la relación AP/LP: ancho del protórax/largo del protórax. La terminología empleada para nominar las estructuras genitales es la dada por Suzuki (16) y Bechyné y Bechyné (9) para los machos y la de Kasap y Crawson (13) para las hembras. Para el estudio de la venación alar se empleó el sistema propuesto por Jolivet (12). Los dibujos se confeccionaron con cámara clara, empleando microscopios óptico y 
estereoscópico. Las fotos se tomaron con la cámara del microscopio electrónico de barrido (JEOL-JMS-T100), perteneciente a la Facultad de Ciencias Naturales y Museo de la Universidad Nacional de La Plata.

\section{Resultados}

\section{Maecolaspis bridarollii (Bechyné) (Figuras 1-21)}

Colaspis bridarollii Bechyné 1949: 507.

Maecolaspis bridarollii Bechyné 1950: 10; 1952: 11; 1953: 217; 1957:239;1958:500.

Macho (Figura 1) Coloración general violácea, bronceada o verde, con brillo metálico; tercio apical de los élitros opaco. Labro, antenas y patas castaño claras, del Vil al XI antenitos castaño más oscuros.

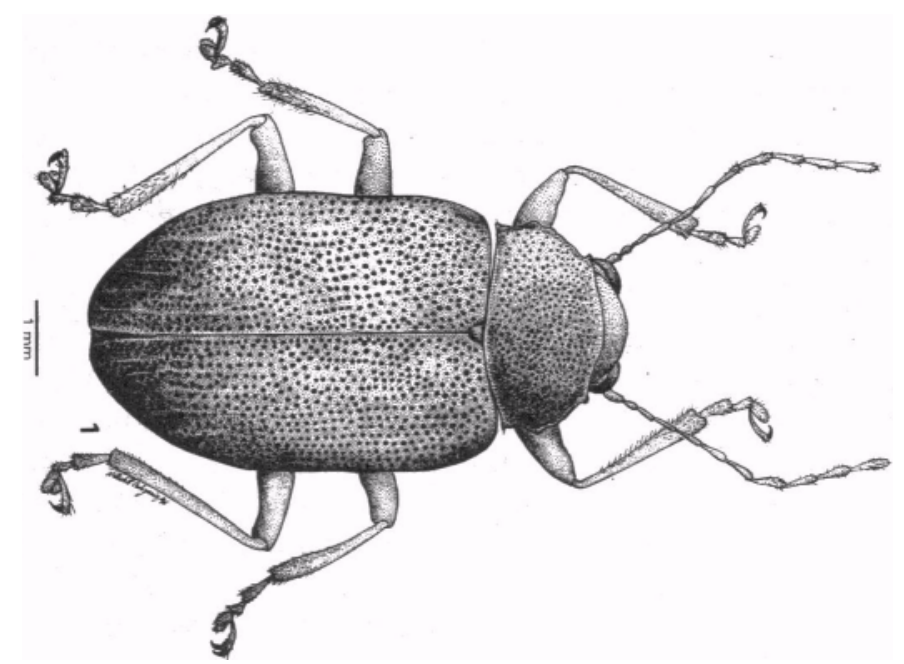

Figura 1. Maecolaspis bridarollii. Habitus del adulto.

Cabeza (Figura 12) Vértex aplanado, sutura coronal poco profunda. Ojos convexos, margen interno recto. Antenas filiformes, delgadas (Figura 2), el II globoso, menos de la mitad del largo del I, del III al VI delgados, subcilíndricos, del Vil al IX deprimidos dorsoventralmente y con una proyección en el extremo apical, $\mathrm{X}$ subcilíndrico, el XI cónico. Extremo apical de los antenitos con 2-4 setas, del VII al XI cubiertos además de pubescencia densa y corta. 
Labro cuadrangular, márgenes laterales redondeados, el apical escotado; con un par de setas centrales cortas y dos pares laterales, las externas más largas. Mandíbulas (Figura 3) robustas, con dos dientes apicales cónicos, divergentes, en vista lateral se observan otros dos robustos y más pequeños ubicados en el margen externo. Sin mola; cóndilo redondeado y bien desarrollado. Maxilas (Figura 4) con el cardo corto y robusto, estipes débilmente dividido, lacinia amplia, más desarrollada que la galea, con setas cortas y numerosas en el extremo apical y un mechón denso de setas finas en el margen lateral. Galea bisegmentada, artejo apical subcilíndrico. Palpo maxilar con el palpífer cónico, el III tres veces el largo del II, el V subcónico. Labio (Figura 5) con el prementón con cuatro pares de setas laterales, lígula subrectangular con dos pares de setas laterales. Palpo labial de tres artejos, el I y II subcilíndricos, el III subcónico. Superficie de la cabeza con puntuación densa y profunda alrededor de los ojos, más fina en el vértex; la superficie es lisa en la base de las antenas.

Tórax (Figura 15) de 1,38 a 1,77 (x: 1,57, n: 12) veces el ancho en el largo; ángulos anteriores redondeados y salientes, los posteriores más pequeños, cada uno con una seta. Margen anterior convexo, el posterior cóncavo en el centro; márgenes laterales redondeados, con un tubérculo pequeño en el centro. Puntuación semejante a la de la cabeza, los puntos en el disco separados, más densos y profundos hacia los márgenes laterales. Escutelo oblongo con la superficie lisa. Proesterno cuadrangular; base del mesoesterno dos veces su extremo apical. Élitros con los húmeros lisos (Figura 17). Puntos más gruesos que en el protórax, no confluentes, ordenado en hileras dobles. En la región próxima al húmero los puntos son poco profundos y aislados. Costillas moderadamente elevadas en el tercio apical y próximas al borde sutural y apenas perceptibles en la región basal; las dos próximas al borde sutural se unen en el extremo apical (Figura 16). Borde elitral con costillas longitudinales que se unen con las apicales (Figura 14), entre ellas dos hileras de puntos paralelos y una intermedia incompleta (Figura 17). Alas (Figura 6) con nerviación típicamente eumolpoide (12), R1 dilatada distalmente, rt suboval, M1 neta, sistema cubito-anal típico, tacha medio-cubital ausente, c bastante larga. Patas delgadas, fémures fusiformes, los posteriores más largos y delgados; tibias acanaladas en el borde externo, escotadas en su extremo apical, con una espina corta en el extremo. Tarsito II dos veces el largo del I, el III con pilosidad corta, densa, setas de tipo espatulado; uñas apendiculadas (Figura 18). 
Abdomen (Figura 7) menos aguzado que en las hembras, el esternito I más largo que el II, margen apical ampliamente cóncavo (Figura 19).

Genitalia. Lóbulo mediano de tipo seudoarticulado (9) (Figura 8). La porción basal, que aloja el ducto espermático, membranosa, y la apical fuertemente esclerotizada. Ápice del lóbulo mediano levemente asimétrico, muy aguzado en vista lateral (Figura 8). Tegmen en forma de $Y$ unida al lóbulo por una membrana. Saco interno con dos escleritos.

Longitud total: 6-7.7 mm; ancho máximo: 3-3.5 mm.

Hembra. De mayor tamaño que el macho. Margen interno de los ojos escotado. Antenas similares a las de los machos (Figura. 9), antenito II más largo que en los machos, globoso, del III al VII subcilíndricos, VIII, IX y X más anchos que en los machos, el XI levemente estrechado en el tercio apical. Tórax (Figura 13) de 1.61 a 1.8 (x: 1.70, n: 12) veces el ancho en el largo, margen anterior recto, el posterior cóncavo, tubérculo del margen lateral más marcada que en el macho, ubicada en el tercio apical. Punteado del disco más denso que en los machos. Costillas elitrales menos elevadas. Tibias posteriores sin espina apical. Abdomen (Fig. 10) aguzado en el extremo, margen apical del último esternito con una escotadura central pequeña (Figura 21).

Genitalia (Figura 11). Las estructuras del aparato reproductor de la hembra corresponde al patrón dado por Kasap y Crawson (13) con la bursa copulatriz desarrollada, sin escleritos. Cápsula de la espermateca larga, ápice aguzado, ducto espermatecal corto. Coxitos con numerosas setas apicales cortas.

Longitud total: 7.3-8 mm; ancho máximo: 4-4.3 mm.

Variación intraespecífica. Bechyné describió la forma con la superficie dorsal violácea bronceada como la forma típica y aquellas de color verde metálico como la aberración aurichalcea. Sin embargo, para el Código de Nomenclatura Zoológica las aberraciones no son válidas por lo que se consideran aquí como una variación de color. 
194

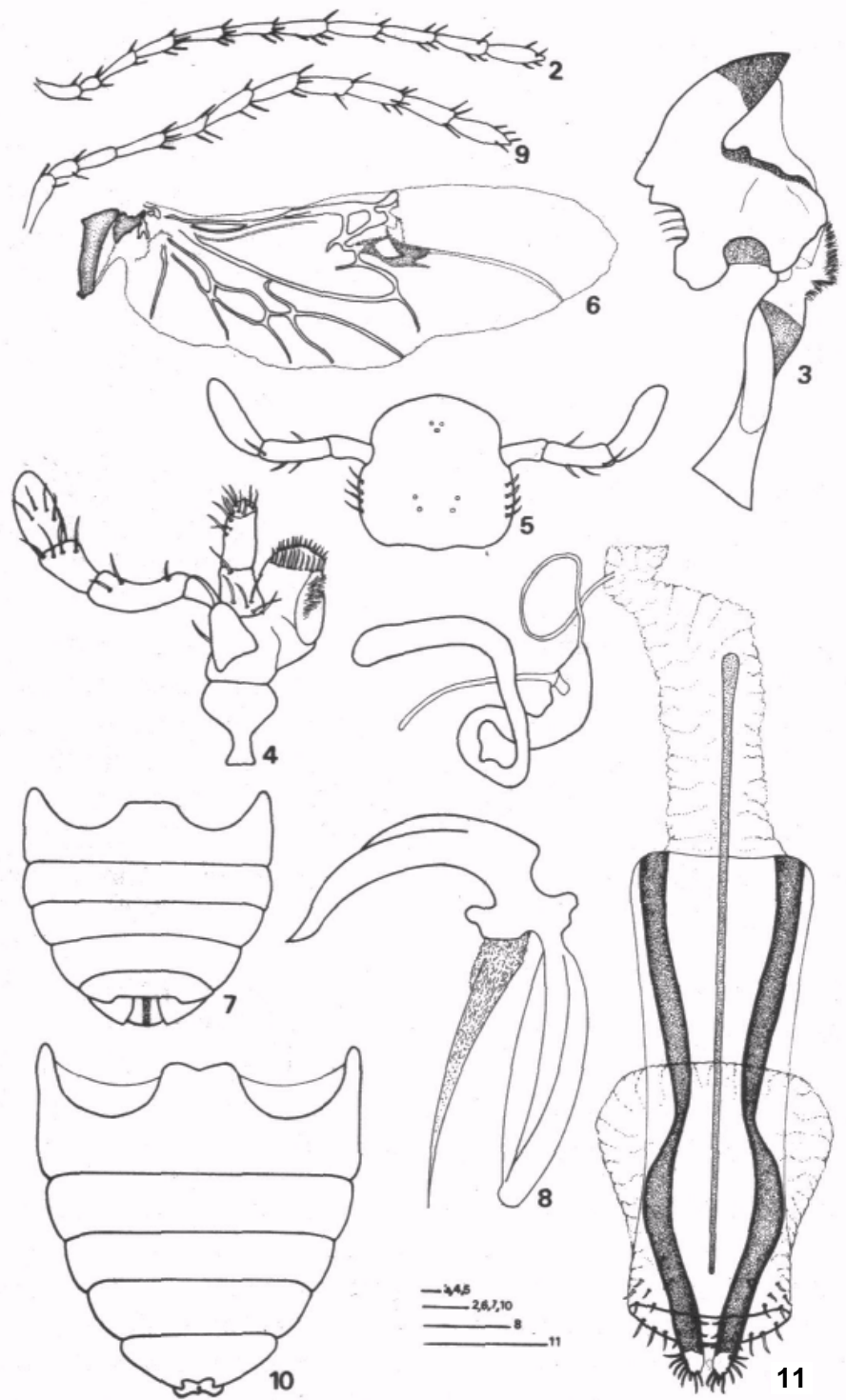

Figuras. 2-11. Maecolaspis bridarollii. 2-8, macho. 2: antena. 3: mandíbula derecha, vista ventral. 4: maxila izquierda, vista dorsal. 5: labio, vista ventral. 6: ala metatorácica. 7: abdomen, vista ventral. 8: lóbulo mediano, vista lateral. 911, hembra. 9: antena. 10: abdomen, vista ventral. 11: genitalia femenina. 


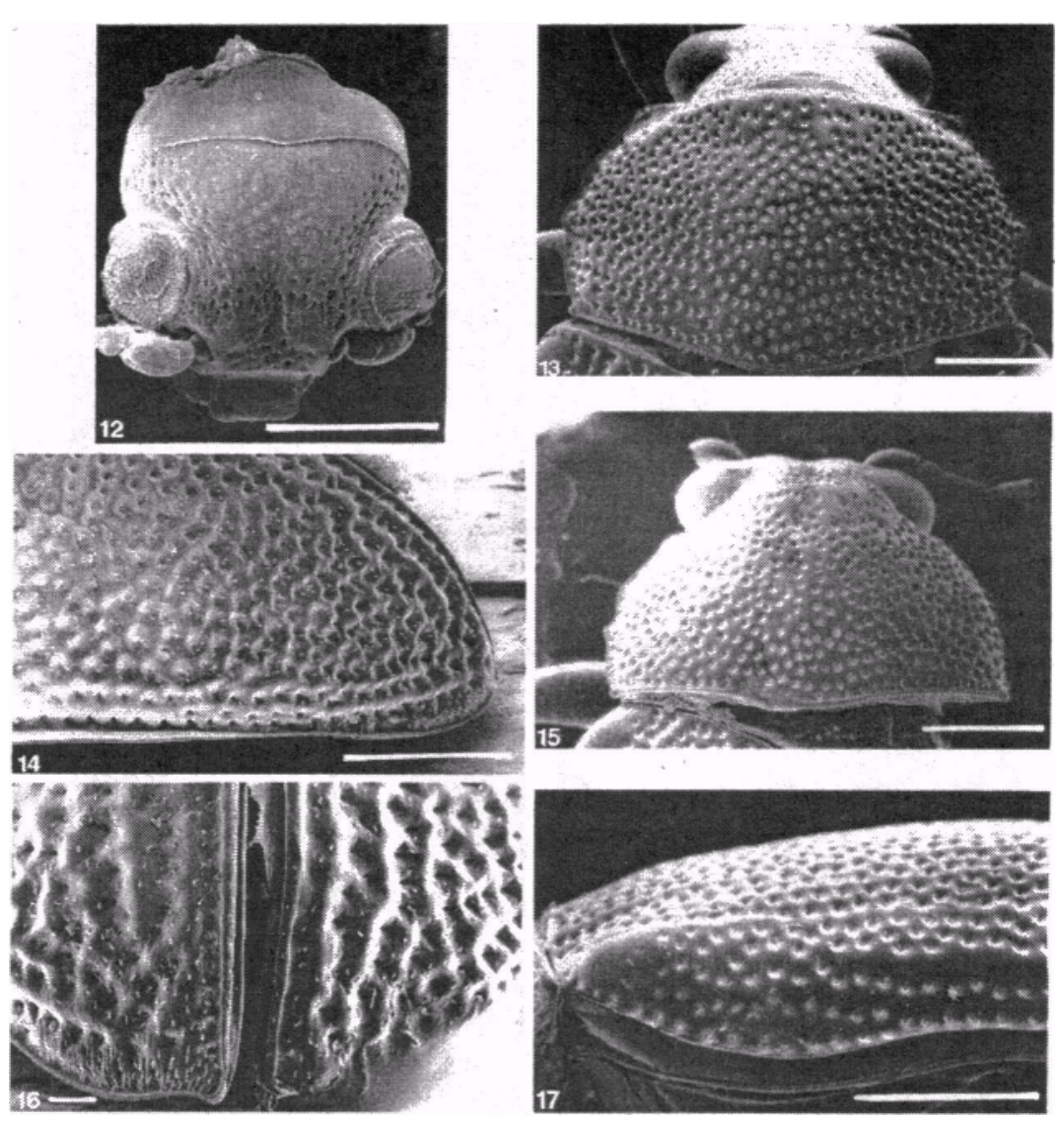

Figuras. 12-17. Maecolaspis bridarollii. 12,14-17, macho. 12: cabeza, vista anterior. 14: élitros, vista posterolateral. 15: protórax, vista dorsal. 16: élitros, vista postero-dorsal. 17: élitros, vista antero-lateral. 13, hembra, protórax, vista dorsal. Escalas 3,4,5, 8:0,1 $\mathrm{mm} ; 2,7,11: 0.5 \mathrm{~mm}$. 


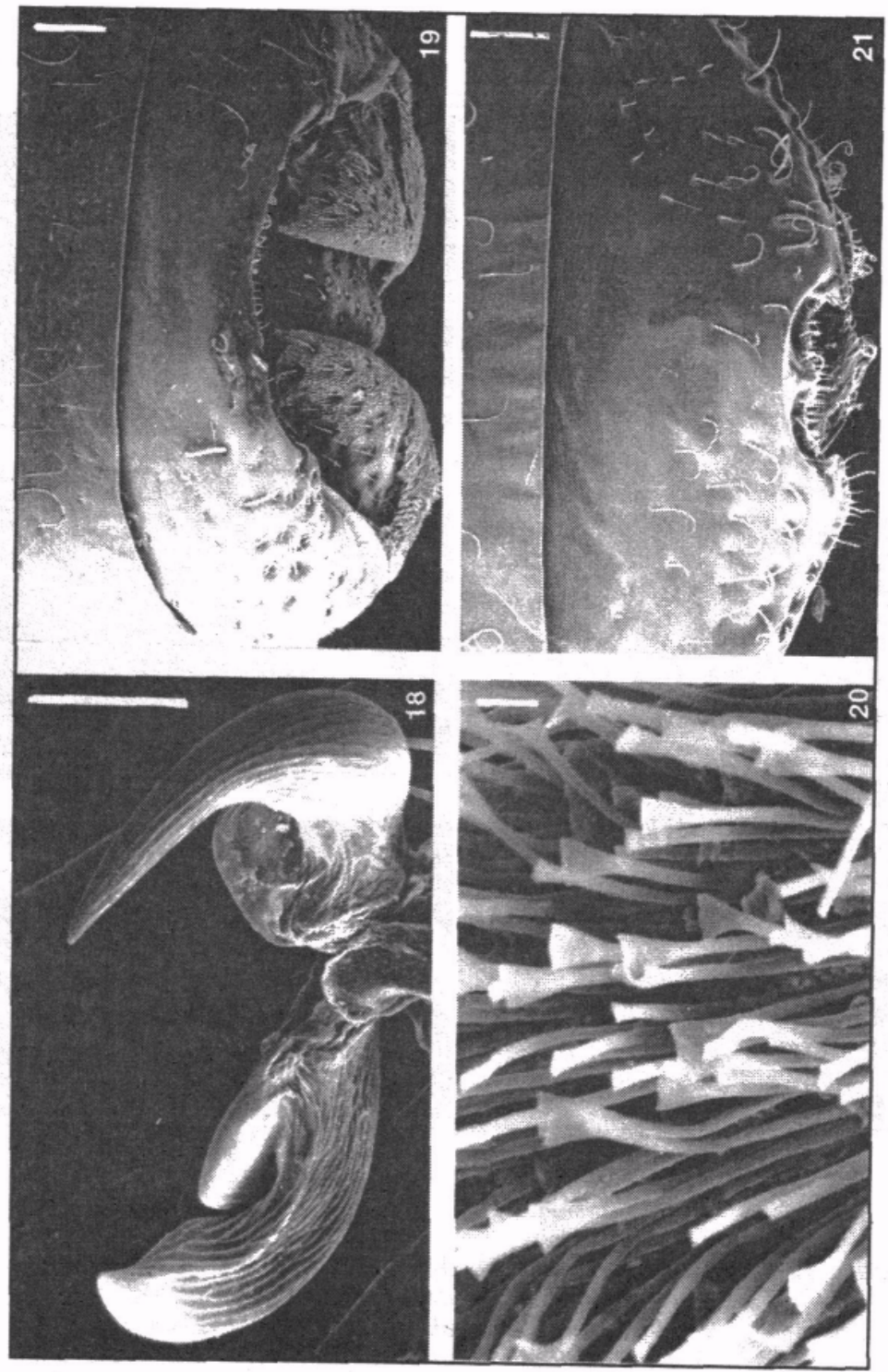

Figuras. 18-21. Maecolaspis bridarollii. 18-20, macho. 18: uñas de las patas posteriores. 19: abdomen, vista dorsal. 20: pata posterior, detalle de las setas tarsales. 21, hembra, abdomen, vista dorsal. Escalas 12-14, 15,17:1 mm; 18: $0.1 \mathrm{~mm} ; 16,19,21: 0$ i mm; 20:0.02 mm. 
Distribución geográfica. Citada para Argentina: Santiago del Estero (2), posteriormente amplió su distribución para Buenos Aires, Córdoba, Entre Ríos, Misiones, Santa Fe y Chaco (4, 5, 6, 7, 8). También está presente en Paraguay, Uruguay y Brasil.

Bionomía. Los adultos se alimentan de la hoja de la planta de papa, dejando perforaciones irregulares en el follaje, y las larvas que han alcanzado mayor desarrollo $(8.0$ a $12.0 \mathrm{~mm}$ ) de los tubérculos. Siguiendo la fenología del cultivo se observó que, los mayores daños se producen a partir de mediados de marzo, cuando las larvas maduras dañan los tubérculos, efectuándoles agujeros profundos que desmejoran su calidad comercial.

Material examinado. ARGENTINA: Formosa: Isla de Oca, $10^{\lambda}$ y $2 \circ \circ, 5-$ I-1938, Denier col. (MLP); 2우, 27-IV, 1939, Denier col. (MLP); Clorinda, 1ㅇ, 10-II-1940, Denier col. (MLP); Herradura, 10, 18-IV-1936, Denier col. (MLP); Mojón de Fierro, $2+$ \% , Denier col. (MLP); sin localidad, 10, Richter col. (MLP). Chaco: Colonia Castelli, 19, 3-V-1936, Denier col. (MLP); Resistencia, 1스-y 2웅, 3-XII-1938, Denier col. (MLP); Puerto Vuelas, $2 \delta^{\lambda}$ y 1ㅇ, 24-XII-1937, Denier col. (MLP). Misiones: Puerto Victoria, 1§, VIII-1946, Bechyné det. (MLP). Corrientes: Manantiales, 10̄, VII-1946, Bechyné det. (MLP); sin localidad, 1ㅇ, Richter col. (MLP). Santiago del Estero: Turena, 1 ㅇ 30-XI-1939, Maldonado col. (MLP); Beltrán, 1ㅇ, I-1942, Bechyné det. (MLP);

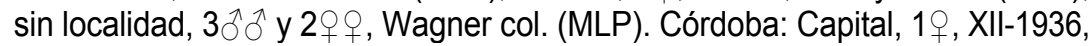
Denier col. (MLP). Santa Fe: Rafaela, 10 y 1ㅇ, 9-II-1932, Denier col. (MLP); Rosario, 19, XII-1928, Denier col. (MLP). Icaño, 1요 Denier col. (MLP). Buenos Aires: La Plata, $2 \hat{\jmath} \hat{\alpha}$ y 8 우우, Bechyné det. (MLP); Balcarce, numerosos $\hat{0} 0^{\hat{y}} \mathrm{y}$ 우, Castillo et al.col (MLP) (INTA). San Fernando, 1, 22XII-1929, Denier col. (MLP); sin localidad, 2o+ 9 , Richter col. (MLP). PARAGUAY: Villa Elisa, XI-1939, Denier col. (MLP); sin localidad, 3 우 (MLP). BRASIL: Matto Grosso, 10 - y 1 우 , Corumbá, Alto Paraguay, $1 \hat{0}-\mathrm{y}$ 1ㅇ, Richter col. (MLP). URUGUAY: Montevideo, 10- y 2우, 24-XII-1907, Tremoleras col. (MLP).

\section{Discusión}

En varias colecciones $M$. bridarollii fue incorrectamente determinada como Colaspis chlorites Erichson. Sin embargo, esta especie se diferencia por ser de color verde brillante, con la superficie elitral cubierta de puntos profundos, ordenados en series longitudinales simétricas y dobles cerca de la sutura y con bordes elitrales débilmente desarrollados. 
M. bridarollii constituye junto con M. scintillifera (Bechyné), M. laplatensis (Bechyné), M. viridissima (Lefevre) y $M$. achardi (Bechyné) un grupo homogéneo, ampliamente distribuido y difícil de identificar individualmente. Las especies comparten similar coloración metálica, protórax densamente punteado, patas enteramente testáceas, puntuación elitral densa y a veces más o menos confluente y ausencia de costillas longitudinales excepto uno o dos laterales.

Más próxima a $M$. scintillifera (Bechyné) por el tamaño pequeño y las costillas perceptibles especialmente en el tercio apical, $M$. bridarollii se diferencia por la coloración opaca del tercio apical de los élitros, la puntuación más débil, la espermateca enrollada, los estilos gruesos y la porción basal del lóbulo mediano más delgada.

Teniendo en cuenta el ciclo de vida de la especie en relación a la fenología del cultivo, la fecha óptima de cosecha sería ni bien finaliza el ciclo del cultivo. De esta manera se evitaría que las larvas, que han alcanzado mayor desarrollo y voracidad, encuentren tubérculos.

\section{Referencias Bibliográficas}

1. Alcázar, J.; Raimundo, S.A. 1985. Nuevos focos de enfermedades y plagas. FAO. Plant Prot. Bull. 33:165.

2. Bechyné, J. 1949. Liste provisoire des Eumolpides de la Republique Argentine et observations diverses sur les Eumolpides de l'Amerique du Sud. (Col. Chrysomeloidea). Acta Zool. Lilloana 8: 457-535.

3. Bechyné, J. 1950. Notes sur les Eumolpides Américains (Col. Phytophaga). Mitt. Münch. Ent. Ges. 40: 245-263.

4. Bechyné, J. 1950. Notes sur les Eumolpides Neotropicaux des collections de l'Institut Poyal des Sciences Naturelles de Belgique. Bull. Inst. r. Sci. nat. Belg. 26 (54): 1-24.

5. Bechyné, J. 1952. Deuxieme note sur les Eumolpides néotropicaux des collections de l'Institut Boyal des Sciences naturelles de Belgique. Bull. Inst. Rech. Sci. Nat. Belgique 28 (38): 1-20.

6. Bechyné, J. 1953. Katalog der néotropischen Eumolpiden (Col. Phytophaga, Chrysomeloidea). Ent. Arb. Mus. G. Frey 4: 26-303. 
7. Bechyné, J. 1957. Eumolpides neotropicaux de la collection du Museo Cívico di Storia Naturale " Giacomo Doria" di Genova (Col. Phytophaga). Ann. Mus. Civ. Stor. Nat. Genova 69: 226-247.

8. Bechyné, J. 1958. Notizen zu den neotropischen Chrysomeloidea (Col. Phytophaga). Ent. Arb. Mus. G. Frey 9: 478-706.

9. Bechyné, J.; Bechyné, B. 1969.(1970). La posición sistemática de Megascelis Chevrolat (Col. Phytophaga). Revista Fac.Agron. Univ. Cent. Venezuela 5 (3): 65-75.

10. Bosq, J. M. 1943. Segunda lista de coleópteros de la República Argentina dañinos a la agricultura. Ing. Agron. 4 (18-22): 7-80.

11. D'Araujo e Silva, A. G., Goncalves, C.R., Monteiro, G. D.et al. 1968. Quarto catalogo dos insectos que vivem ñas plantas do Brasil, sus parásitos e predatores. Ministerio da Agricultura, Departamento de Defesa e Inspecao Agropecuaria, Servicio de Defesa Sanitaria Vegetal, Laboratorio Central de Patología Vegetal, Rio de Janeiro, G. B. Brasil.

12. Jolivet, P. 1957. Recherches sur I aile des Chrysomeloidea (Coleoptera). 2eme serie. Mem. Inst. Rech. Sci. Nat. Belgique 58: 1-193.

13. Kasap, H; Crowson, R. A. 1980. The female reproductive organs of Bruchidae and Chrysomelidae (Coleóptera). Turk. Bitk. Oruma Derg. 4 (2): 85-102.

14. Savini, V. 1993. Nuevas contribuciones al género Maecolaspis (Coleoptera, Chrysomelidae, Eumolpinae). Bol. Entomol. Venezuela N. S. 8 (2): $151-155$.

15. Secretaría de Agricultura Ganadería y Pesca (SAG y P). 1995. Dirección de Información y Sistemas. Informe Técnico. 7 p.

16. Suzuki, K. 1988. Comparativo morphology of the internal reproductive system of the Chrysomelidae (Coleoptera). En: P. Jolivet, E. Petitpierre \& T. H. Hsiao (eds.). Biology of Chrysomelidae. Kluwer Academic Publishers. London. 615 p. 